\title{
Correction to: Applied Head and Neck Anatomy for the Facial Cosmetic Surgeon
}

Elie M. Ferneini, Michael T. Goupil, Margaret A. McNulty, and Christine E. Niekrash

\section{Correction to:}

E. M. Ferneini et al. (eds.), Applied Head and Neck Anatomy for the Facial Cosmetic Surgeon, https://doi.org/10.1007/978-3-030-57931-9

The book was inadvertently published with an incorrect figure legend in Fig. 14.2 of Chap. 14 and the figure caption has now been corrected in this version.

In addition, Fig. 20.2c of Chap. 20 has a watermark over the image. The watermark has now been removed and the corrected figure is available.

The updated version of this chapter can be found at https://doi.org/10.1007/978-3-030-57931-9_14 https://doi.org/10.1007/978-3-030-57931-9_20 